\title{
Dominating Set based Connectivity Maintenance in Wireless Sensor Networks
}

\author{
Joy Winston, PhD \\ King Saud University \\ Riyadh \\ Kingdom of Saudi Arabia
}

\author{
Aadil Ahmad Shah \\ King Saud University \\ Riyadh \\ Kingdom of Saudi Arabia
}

\begin{abstract}
The ability to report the sensed data to base station is called connectivity. The sensor network remains connected so that the information collected by sensor nodes can be transmitted back to the base station. Connectivity depends mainly on the existence of link. It is affected by changes in network topology due to mobility, the failure of sensor nodes and attacks and so on. Therefore, Coverage alone in WSN is not sufficient. Therefore, WSN must satisfy both Coverage and Connectivity required by applications simultaneously.in this we proposed RC-CDS algorithm to provide coverage and connectivity in WSN. We compared the proposed algorithm with the existing algorithms and we find that it outperforms well.
\end{abstract}

\section{Keywords}

Connectivity, coverage, energy, WSN, security

\section{INTRODUCTION}

We The objective of this chapter is to make coverage maintenance for data collection and extend the lifetime of the sensor nodes as long as possible by selecting only a subset of nodes to be on-duty state and keeping the remaining nodes in off-duty state. Besides coverage, connectivity is another fundamental issue in a WSN. The connectivity requirement ensures that any active sensor in the network is able to communicate to the monitoring station at all times using relay sensor nodes, if necessary. A suitable connectivity is highly required in order to achieve robust and smooth communication in a WSN. The communication range is twice of sensing range is the sufficient condition to ensure that complete coverage preservation implies connectivity among active nodes if the original network topology is considered [1]. For example in Figure 6.1, the node $\mathrm{x}$ is the source node, node $\mathrm{y}$ is the destination node, $\mathrm{r}_{\mathrm{T}}$ is the transmission radius and $r_{S}$ is the sensing radius of the source node. Nodes S1, S2, $\mathrm{S} 3$ are the intermediate nodes. The source node transfers the packet to the destination node via intermediate nodes by sensing the transmission radius of each sensor nodes.

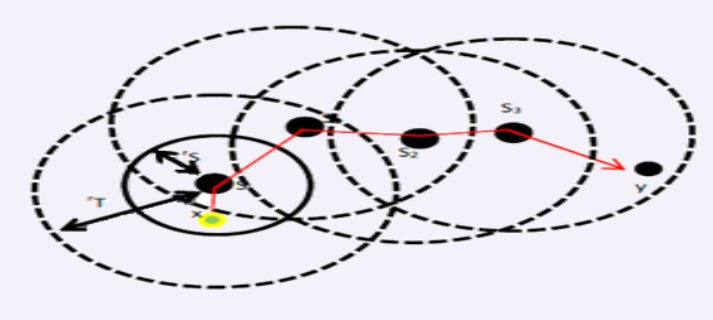

Figure .1 An example of sensor's coverage and connectivity
Network connectivity maintenance in failure prone environment has received more attention in the recent years. Unfortunately due to hostile environment there is need of some other active nodes i.e. backbone nodes which can compensate the failure of the nodes. Using a virtual backbone infrastructure which is one kind of hierarchical methods is an efficient way to lower energy consumption in routing and performing data aggregation. Using this virtual backbone, a sender can send messages to its neighboring dominator. Then along the backbone nodes, the messages are sent to the dominator closest to the receiver. Finally, the messages are delivered to the receiver [2]. A Dominating Set (DS) of a graph is a subset of nodes such that each node in the graph is either in the subset or adjacent to at least one node in that subset.

A Connected Dominating Set (CDS) is a DS, which induces a connected sub graph. A CDS is a good candidate of a virtual backbone for wireless networks, because any node in the network is less than 1-hop away from a CDS node. Only the backbone nodes are responsible for relaying messages for the network. The non-backbone nodes can thus turn off their communication module to save energy when they have no data to be transmitted out. One objective for constructing the backbone is to minimize the size of a backbone (i.e., the number of backbone nodes). Unfortunately computing a Minimal CDS (denoted by MCDS) of a Unit disk graph has been proved to be NP-hard [3]. When comes to network coverage, given a randomly and densely deployed wireless sensor network, it is desirable to have sensors autonomously schedule their duty cycles while satisfying the sensing coverage degree. The problem is called coverage maintenance.

The connectivity maintenance provides a network backbone to support network-wide routing functionality. It supports application specific sensing queries and data gathering of the sensors. It is proposed to induce the connected dominating set to form a virtual backbone in the networks to maintain network connectivity. To cut down communication overhead at best, it is essential to find a minimum connected dominating set of the network graph. An MCDS satisfies: (1) each node is either a backbone node or is one-hop connected to a backbone node. (2) The backbone nodes are connected. After the backbone construction, the backbone nodes will turn on the RF units for connectivity need, and the non-backbone nodes will turn off the RF units to save energy. A CDS only preserves 1-connectivity. However, to achieve robustness, kconnectivity should be guaranteed, where k-connectivity requires that between any pair of nodes in a CDS there exist at least $\mathrm{k}$ different paths. With k-connectivity, communication may not be disrupted even when up to $\mathrm{k}-1$ paths fail. $\mathrm{K}$ connected virtual backbones also provide multi-path 
redundancy for load balancing or transmission error tolerance [4].

\section{DOMINATING SET THEORY}

Domination is an area in graph theory with an extensive research activity such as optimization, design and analysis of communication networks, computational complexity and algorithm design. The historical roots of domination are said to be the problem of determining the minimum number of queens which are necessary to cover an $\mathrm{n} x \mathrm{n}$ chessboard. The concept of a dominating set occurs in a variety of problems. A number of these problems are motivated by communication network problems, for example in computer networks; it is desirable to use a DS in order to maintain a hierarchical structure in which the members of the DS provide good service to their neighbors. Since the connectivity of a WSN is constantly changing, routing protocols for WSN differ significantly from the standard routing schemes which are used in wired networks. One effective way to maintain the connectivity is by using a DS, where only the dominating nodes of the set will act as routers and all the other nodes communicate through a neighboring node in the DS [5].

A DS for a graph is a set of vertices which, along with their neighbors, constitute all the vertices in the graph. An unweighted graph $\mathrm{G}=(\mathrm{V}, \mathrm{E})$ is used to represent a WSN, where $\mathrm{V}$ represents a set of wireless sensor nodes, and $\mathrm{E}$ represents a set of edges. For any vertex $\mathrm{x} \in \mathrm{V}$, the neighborhood of $\mathrm{x}$ is denoted by $\mathrm{N}(\mathrm{x})$, as the set of all vertices is adjacent to $\mathrm{x}$. The closed neighborhood of $\mathrm{x}$, denoted by $\mathrm{N}[\mathrm{x}]$, is the set $\mathrm{N}(\mathrm{x}) \mathrm{U}\{\mathrm{x}\}$. For a set of vertices $\mathrm{S}, \mathrm{N}(\mathrm{S})$ as the union of $\mathrm{N}(\mathrm{x})$ for all $x \in S$, and $N[S]=N(S) U S$. The degree of a vertex is the size of its neighborhoods. The maximum degree of a graph $\mathrm{G}$ is denoted by $\Delta(\mathrm{G})$, and the minimum degree is denoted by $\delta(\mathrm{G})$.

Mathematically, a DS is defined as a set of vertices $S$ in a graph $\mathrm{G}(\mathrm{V}, \mathrm{E})$ if every vertex $v \in \mathrm{V}$ is either an element of $\mathrm{S}$ or is adjacent to an element of $\mathrm{S}$. A set $\mathrm{S}$ of vertices in a graph $\mathrm{G}(\mathrm{V}, \mathrm{E})$ is called a total dominating set if every vertex $\mathrm{v} \in \mathrm{V}$ is adjacent to an element of $\mathrm{S}$ and the domination number of a graph $\mathrm{G}$ denoted by $\gamma(\mathrm{G})$ is the minimum cardinality of a dominating set in G. A CDS is a dominating set, which induces a connected sub graph of $\mathrm{G}$ (Jeremy Blum et al 2005). A network is called CDS only if it (i) should be connected, (ii) should be reasonably small, (iii) should contain shortest paths and (iv) should consist of nodes which possess enough energy resources. The principle of forming a CDS from the group of a network has been explained below using a small illustration.

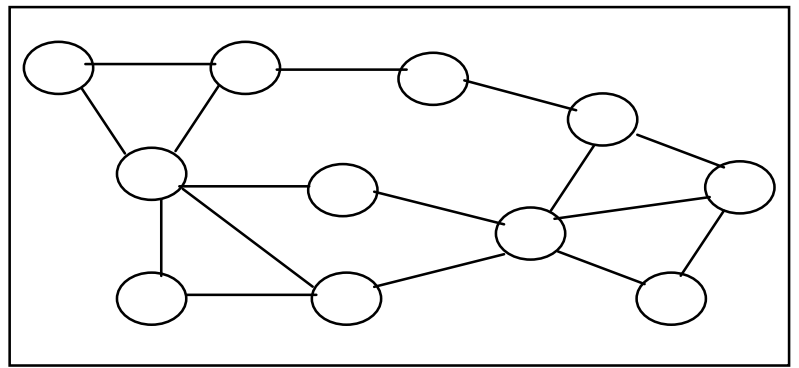

Figure 2 Typical WSN

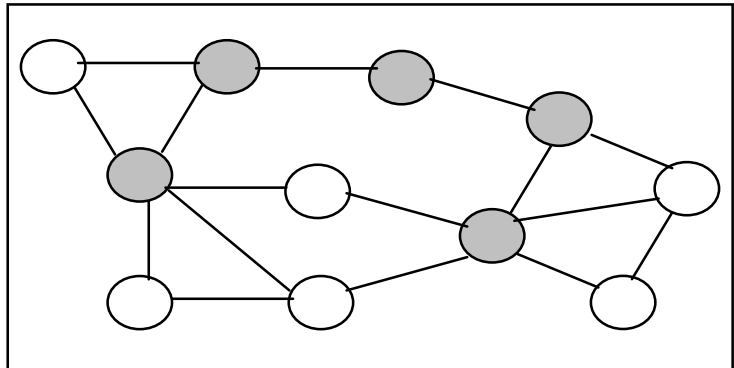

Figure 3 Illustrating DS

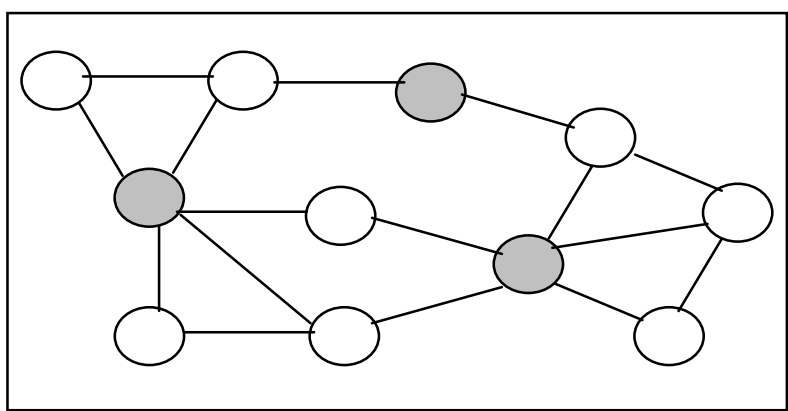

Figure 4 Illustrating CDS

Figure 2 shows a typical WSN. Figure 3 illustrates the DS formation for the network. There are three dominating nodes in the set, which are marked in gray, and Figure 4 is the CDS network for the original network shown in Figure 2. This CDS contains five dominating nodes, which are also marked in gray. Well-known algorithms for constructing a CDS normally consider the number of nodes (cardinality) as the sole criterion which emphasizes the fact that a smaller dominating set can produce better connectivity. To reduce the communication overhead further, to increase the convergence speed and to maintain minimum connectivity management, it is desirable to construct a Minimum Connected Dominating Set (MCDS) for a given set of nodes. MCDS is the CDS with a minimum cardinality. MCDS helps to decrease the control overhead, and it also helps to update the topology only for a small subset of nodes. The construction of the MCDS is a well-known problem in graph theory. A Weakly Connected Dominating Set (WCDS) $\mathrm{S}$ is a dominating set $\mathrm{N}[\mathrm{S}]$ that induces a connected subgraph of $\mathrm{G}$. In other words, the subgraph weakly induced by $\mathrm{S}$ is the graph induced by the vertex set containing $S$ and its neighbors [6]. Given a connected graph $\mathrm{G}$, all the dominating sets of $\mathrm{G}$ are weakly connected.

Unfortunately, the dominating set problem has been proved to be NP-Hard. Computing a minimum WCDS is also NP-Hard. For broadcasting, multicasting, routing and related tasks, it is beneficial to construct a small sized (reduced number of active nodes) connected network that represents the backbone of the entire network. Many works seek an MCDS in a Unit Disk Graph (UDG). The communication range of a node in a WSN is typically modeled as a disk, centered at the node with a radius equal to the transmission range of the radio. When the transmission range is fixed for all the nodes, the network has the property of a unit disk graph, where an edge exists if and only if two nodes have an inter-nodal distance less than or equal to 1 unit [7]. 


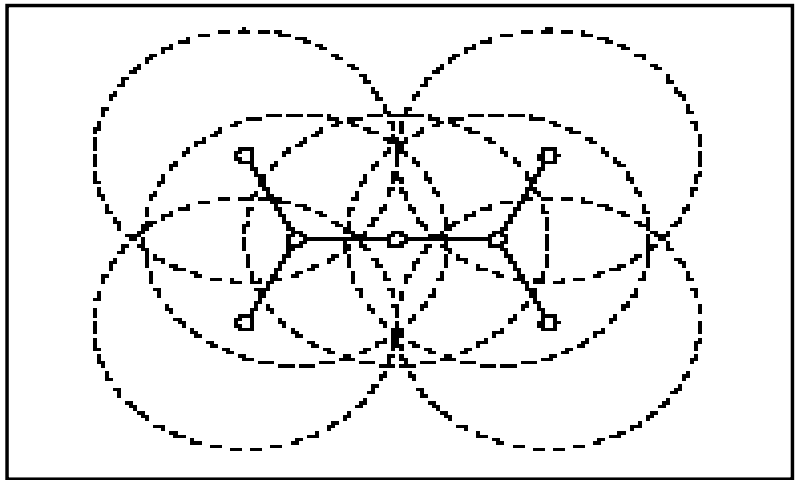

Figure 5 Illustrating a UDG

In Figure 5, a UDG has been constructed from seven nodes; the small dots represent nodes. The dotted circles represent the transmission radius of each node. If the transmission ranges of two nodes overlap, then a connection is established between these nodes; otherwise connection establishing is not possible. A CDS can serve as a virtual backbone to any network. The virtual backbone has to be designed in such a way that the nodes not present in the backbone are within the transmission range of some other nodes in the backbone. The searching space for a route is reduced for nodes in the backbone. The nodes in the subset act as backbones used for routing by the rest of the nodes not in this subset. CDS is mainly used in energy efficient routing by putting nonbackbone nodes into an inactive state; the energy consumption is greatly reduced, while network connectivity is still maintained by the backbone nodes [8]

The design goals of any application dictate the selection from the different techniques for creating a virtual network structure. Depending on the needs of the application, either a WCDS or a CDS will be selected. While designing a CDS, the requirements of stability will affect the size and characteristics of the dominating set as well as the node selection methodology. Also, the selection of a WCDS or a CDS depends on the communication requirements between nodes within the DS. Mainly, routing-based applications tend to rely on CDS, since messages must be forwarded along a backbone (Ceronmani Sharmila1 and George 2014). However, if the network topology is highly unstable, a WCDS may be a better choice since its smaller size makes it easier to maintain. Likewise, while intra-cluster coordination functions might be managed within a WCDS, inter-cluster coordination is probably more easily handled by a CDS. So, if adjacent clusters are using orthogonal codes or different frequency bands, a WCDS could manage media access within each cluster. However, for system-wide media access coordination, a CDS may be more efficient since it includes nodes needed for cluster head communication.

Moreover, if a network has a high number of mobile nodes by the time the dominating set has been constructed, the network topology may get changed and the performance of the algorithm may not be as good as that of a static dominating set. In addition to performance considerations, the stability requirements of the dominating set are also crucial for many applications that require long-lasting dominating sets. A user may seek to reduce the rate of change in a CDS topology. Alternatively, others may seek to increase the duration that a node is saved as a dominating node in the dominating set. Depending upon the stability factor, different techniques can be employed. If one seeks to promote the stability of the CDS, then it selects low mobility nodes as members [9]. However, if one also seeks to promote the stability between the nodes and members of the CDS, then one must construct a DS based on the predictions of the relative mobility between nodes. Furthermore, these predictions are domain-specific predictions. So it is unsuitable for domains where mobility is severe. Many CDS construction algorithms achieve greater stability by relaxing the constraints on the number of elements in a dominating set that are within radio range of each other.

Even though it is proved that centralized CDS Construction algorithms support many WSN applications effectively, they face some shortfalls while being applied for some mission critical real-time applications like tracking and monitoring. Non-classical issues like failure of nodes in CDS, practical node mobility scenario, short coming in the expected lifetime compared with actual lifetime, unexpected network partitioning, failures in network due to lack of global knowledge, drawbacks in the construction of paths from the root node to the leaf node direction and regular issues in shorter lifetime, delay, communication overhead, performance and stability are major issues that need attention [10].

\section{PROPOSED SCHEME}

In this chapter a Reduced Cardinality Connected Dominating Set (RC-CDS) algorithm is proposed to maintain connectivity by dominating nodes to be on-duty state and keeping the remaining nodes in off-duty state. It consists of four phases namely

i) Computation of Maximal Independent Set (MIS)

ii) Construction of Connected Dominating Set (CDS)

iii) Reduce the size of CDS

iv) Node Self-Scheduling Phase

\subsection{Computation of Maximal Independent Set (MIS)}

Maximal Independent Set (MIS) is an independent set that is not a subset of any other independent set. A MIS is also a dominating set in the graph and every dominating set that is independent must be maximal independent so maximal independent sets are also called independent dominating sets. In this phase, an algorithm is based on the construction of maximal independent set of the given network graph.

Step 1: Arrange all the vertices of the network graph ' $G$ ' in some order $\mathrm{V}=\mathrm{v}_{1}, \mathrm{v}_{2}, \mathrm{v}_{3}, \ldots \ldots \ldots . \mathrm{v}_{\mathrm{n}}$.

Step 2: Select a vertex $\mathrm{v}_{1}$ from $\mathrm{V}$ and create a set $\mathrm{S}_{1}=\left\{\mathrm{v}_{1}\right\}$.

Step 3: Select vertex $v_{2}$. If $v_{2}$ is not adjacent to $v_{1}$, then put it in set $S_{1} . S_{1} \rightarrow S_{1} U\left\{v_{2}\right\}$ else create a new set $S_{2}=\left\{v_{2}\right\}$.

Let $\mathrm{v}_{1}, \mathrm{v}_{2}, \mathrm{v}_{3}, \ldots \ldots \mathrm{v}_{\mathrm{i}-1}$ vertices in ' $\mathrm{V}$ ' have been selected and included in the sets $S_{1}, S_{2}, S_{3}, \ldots \ldots S_{m}$.

Step 4: Select the vertex $v_{i}$ and find $N\left(v_{i}\right)$.

if $\mathrm{N}\left(\mathrm{v}_{\mathrm{i}}\right) \mathrm{N}\left(\mathrm{v}_{\mathrm{i}}\right) \cap \mathrm{S}_{\mathrm{j}}=\phi$ for $j \in\{1,2, \ldots \ldots . m\}$ then $\mathrm{S}_{\mathrm{j}}$ $\rightarrow \mathrm{S}_{\mathrm{j}} \mathrm{U}\left\{\mathrm{v}_{\mathrm{i}}\right\}$.

Else if $\mathrm{N}\left(\mathrm{v}_{\mathrm{i}}\right) \cap \mathrm{S}_{\mathrm{j}}=\phi$ for $j \in\{1,2, \ldots \ldots . m\}$ then $\mathrm{S}_{\mathrm{j}+1}=\left\{\mathrm{v}_{\mathrm{i}}\right\}$.

Step 5: Repeat Step 4 for all vertices $V_{i}$ 's untill the $i$ is finished. Hence, independent sets $\mathrm{S}_{1}, \mathrm{~S}_{2}, \mathrm{~S}_{3}, \ldots \ldots \ldots \mathrm{S}_{\mathrm{m}}$ are obtained.

Step 6: Find an access point (AP) or sink node 'A' from the network graph ' $\mathrm{G}$ '. 
Step 7: Remove an access point or sink node 'A' from the independent sets of the network graph.

Step 8: Find the independent sets ' $\mathrm{S}_{\max }$ ' which include maximum number of vertices from the vertex set of the network graph.

Therefore ' $\mathrm{S}_{\max }$ ' is the maximal independent set of the network graph ' $G$ '. MIS $=S_{\max }$. Thus, maximal independent set 'MIS' are obtained.

\subsection{Construction of Connected Dominating Set (CDS)}

In this phase, the dominating set is created which is based on the maximal independent set of the given network graph that were created in previous phase, therefore all the data is transmitted to the base station using this dominating set. By the definition of dominating set, the maximal independent set is considered as the dominating set. Therefore

$\mathrm{DS}=\mathrm{MIS}=\mathrm{S}_{\max }$. The input and output of this algorithm are:

Input: Input is Dominating Set (DS)

Output: Connected Dominating Set (CDS)

It consists of the following steps

Step 1: Remove the dominating set vertices from the vertex set ' $V$ ' of the network graph ' $G$ '. Therefore $V$ ' $=V-S_{\text {max }}$

\section{Step 2: Dominating Tree Construction}

- Let $\mathrm{v} \in \mathrm{V}$ ' be the largest degree node. Include $\mathrm{v}$ to empty tree $\mathrm{T}$.

- For all nodes w adjacent to $\mathrm{v}$, choose largest degree node. Add edge $(\mathrm{v}, \mathrm{w})$ to $\mathrm{T}$.

- If two or more nodes like $\mathrm{w}_{1}, \mathrm{w}_{2} \ldots \ldots, \mathrm{w}_{\mathrm{n}}$ has largest degree add edge $\left(\mathrm{v}, \mathrm{w}_{1}\right),\left(\mathrm{v}, \mathrm{w}_{2}\right), \ldots \ldots \ldots \ldots\left(\mathrm{v}, \mathrm{w}_{\mathrm{n}}\right)$ to T.

- Repeat the above steps until all the nodes V' were present in the tree $\mathrm{T}$.

- After constructing the partial dominating tree remove all leaf nodes present in the tree. Now the tree is called dominating tree.

Step 3: CDS-Queue construction

- Queue Q=Null

- Based on the dominating tree construction, classify the nodes in the tree according to the level of the tree.

- From lower level to upper level of tree $\ln , \ln -1, \ldots$ 10. Add elements in $\ln$ to $Q$, until all elements in various levels to $\mathrm{Q}$.

Step 4: Find the connectors $\left(\mathrm{c}_{\mathrm{i}}\right)$ for the dominating set.

After constructing the dominating tree, some node is not truly dominant ie they are connectors. To find those nodes, we give some criteria.

- While there is at least one element in Q \{

- Let $w$ be the element with one unconnected dominant node and one regular node that has maximum degree.
- Remove the element $\mathrm{w}$ from the queue and delete that node from the tree, since the node is considered as connector.

- The element is added to the connector set C

Step 5: Repeat Step 4 until all the connectors is find out.

Step 6: After finding all the connectors of the network graph ' $\mathrm{G}$ '. The user selects the connectors $\left(\mathrm{c}_{\mathrm{i}}\right)$ according to its requirement for the transmission. Thus connectors $(\mathrm{C})$ are obtained.

\subsection{Reduce the size of CDS}

To further reduce the size of CDS, it is observed that what is more related to the size of CDS is the node degree. According to the proposed algorithm the Step 4 in the previous phase still removes some nodes in CDS. Because they act as dominant node but originally they are the connectors.

\subsubsection{Node Self-Scheduling Phase}

A consequence of the above said phases is that if a node is selected as dominating node for the entire transmission period, this node will soon face a shortfall of energy, and as a result the network will die. That is why a dynamic selection of dominating nodes is introduced. The selection is done based on the energy level of nodes after every transmission in the network. For the initial round, the dominating nodes can be selected with the use of the RC-CDS algorithm, and for the succeeding rounds, those nodes are not necessary to act as dominating nodes. A condition is imposed here that only those nodes which have a higher energy level than the threshold energy level can act as dominating nodes for the succeeding rounds. If more than one node has an equal energy level, then the highest degree node is selected as the dominating node. The energy consumption of nodes is equally distributed because most nodes in the network can share the role of dominating node, which in turn, balances the load of the routes in the network. In sum, the protocol increases the lifetime of the network by keeping the nodes alive for a long time.

\subsubsection{Theoretical Analysis}

Lemma 1 : The spanning tree $T$ generated from this algorithm represents a connected graph $R$.

Proof: Let $\mathrm{G}$ be a connected graph. Let $\mathrm{T}$ be a minimal connected-spanning subgraph of $\mathrm{G}$. Then for any line $\mathrm{X}$ of $\mathrm{T}$, $\mathrm{T}-\mathrm{X}$ is disconnected and hence $\mathrm{X}$ is a bridge of $\mathrm{T}$. Hence $\mathrm{T}$ is acyclic. Further $\mathrm{T}$ is connected and hence is a tree.

Lemma 2 : The time complexity of RC-CDS is $O(n)$.

Proof: In order to guarantee that the network is connected, the transmission radius of should satisfy the following formula.

$$
r \geq \frac{\sqrt{2}}{2} M \sqrt{\frac{1}{\mathrm{n}} \log \left(\frac{n}{\delta}\right)}
$$

Where $\delta$ represents the probability that the network is not connected. In this algorithm, the most time-consuming operation is the step 4 in CDS construction. In the worst case, the statement cycles by $\mathrm{n}$ times, so the algorithm's time complexity is $\mathrm{O}(\mathrm{n})$.

\section{SIMULATION AND RESULTS 4.1 Simulation study}

This work is implemented over the Network Simulator 2.32. A WSN of $\mathrm{n}$ nodes is randomly deployed into $500 \times 500 \mathrm{~m}^{2}$ 
area of interest where 50 vertex sets are generated for each case. The simulation results are studied by varying the network size from 100 to 600 and by varying the number of rounds from 200 to 1000 . All sensor nodes are assigned to have the same hardware and transmission power. The results are simulated over different simulation topologies. The parameters used in simulation are tabulated in Table 1.

Table 1.Parameter settings for simulation

\begin{tabular}{|l|l|}
\hline \multicolumn{1}{|c|}{ Parameter } & value \\
\hline Deployment Area & $500 \mathrm{~m} \times 500 \mathrm{~m}$ \\
\hline Number of nodes & $100-600$ \\
\hline Simulation time & $200 \mathrm{~s}: 1000 \mathrm{~s}$ \\
\hline Initial energy of each node & 2 joules \\
\hline Sensing Range & $10: 50 \mathrm{~m}$ \\
\hline Communication Range & $20 \mathrm{~m}$ \\
\hline
\end{tabular}

\subsection{Algorithms used for Comparison}

The performance of RC-CDS is evaluated by comparing with related schemes CCH-MCDS [11], E-MCDS [12] and IPCDS [13].

Collaborative Cover Heuristic Minimum Connected Dominating Set (CCH-MCDS) is a heuristic technique for construction of minimum connected dominating set in wireless sensor networks. They are using two principles: 1) domatic number of a connected graph is at least two and 2) optimal substructure is defined as subset of independent dominator preferably with a common connector. They obtain a partial Steiner tree during the construction of the independent set. A final post-processing step identifies the Steiner nodes in the formation of Steiner tree for the independent set of graph. They show that the collaborative cover heuristics are better than degree-based heuristics in identifying independent set and Steiner tree. While their distributed approximation CDS algorithm achieves the performance ratio of $(4.8+\ln 5)$ opt +1.2 , where opt is the size of any optimal CDS, they also show that the collaborative cover heuristic is able to give a marginally better bound when the distribution of sensor nodes is uniform permitting identification of the optimal substructures. Also, they show that the message complexity of their algorithm is $\mathrm{O}\left(\mathrm{n}|\Delta|^{2}\right), \Delta$ being the maximum degree of a node in graph, and the time complexity is $\mathrm{O}(\mathrm{n})$.

Energy-efficient MCDS (E-MCDS) is an efficient approximation MCDS construction algorithm which explicitly takes energy consumption into account. E-MCDS contains two stages: the CDS construction stage and the pruning stage. The constructed CDS is approximately composed of two independent sets. The performance ratio of E-MCDS is analyzed in both unit disk graph and disk graphs with bidirectional links, being 9.33opt and 17.33opt respectively. By simulation, the size of CDS constructed by EMCDS is smaller than that of the compared algorithms, and the energy efficient of E-MCDS is better than that of the compared algorithm LEACH. The message complexity of EMCDS is $\mathrm{O}(\mathrm{n})$. The simulation results show that E-MCDS performs well both in terms of the size of CDS constructed and the energy efficiency.
IPCDS is a virtual backbone construction algorithm based on connected dominating set in wireless sensor networks. The algorithm gives priority to more energy and closer nodes in the choice of backbone nodes, which makes network life longer and delay smaller. This algorithm prefers more energy, large RSSI (Received Signal Strength Index) nodes to become backbone nodes. It uses the staining and markers methods to solve the maximum independent set and connected dominating set, and uses the pruning rule to further reduce the connected dominating set. Theoretical analysis shows that the connected dominating set generated by the IPCDS algorithm is up to $7.6 o p t+1.4$, the message complexity and the time complexity are $\mathrm{O}(\mathrm{n})$.

\subsection{Results and Discussion}

In this section, the simulation results of the proposed RC-CDS construction algorithm are analysed. In order to analyse the performance of the proposed algorithm RC-CDS, its performance is compared with CCH-MCDS, E-MCDS and IPCDS algorithms. The performance of RC-CDS is analysed based on the parameters like total cost of the CDS, size of $\mathrm{CDS}$, average risk factor, interference and coverage.

Total cost of the CDS: This metric defines the total energy cost consumed by nodes involving in the process of construction of connected dominating set.

Size of CDS: The nodes in the CDS are called dominators (backbone nodes). It defines the size of the CDS.

Average Risk factor: The average risk factor of a dominating tree $\mathrm{T}$, denoted by avg-risk(T) is the average value of $\operatorname{cov}(\mathrm{x})$ where $\mathrm{x}$ is a child of the root node. $\operatorname{cov}(\mathrm{x})$ denotes the number of nodes that can be reached only through node $\mathrm{x}$ when the dominating tree is used to broadcast a message, and $n$ is the number of nodes in the dominating tree. It is a good measure that the risk factor should reflect the extent of the vulnerability of the CDS when some members of the dominating set become inactive.

Interference: Let the two nodes incident to edge $e_{1}$ be ' $u$ ' and ' $v$ ' and the nodes incident to edge $e_{2}$ be ' $x$ ' and ' $y$ '. The interference of a node ' $u$ ' is a disk centered at ' $u$ ' with radius of R. if node ' $u$ ' or node ' $v$ ' are covered by one of the interference disks of node $\mathrm{x}$ and $\mathrm{y}$ then the edge $\mathrm{e}_{1}$ is interference with edge $\mathrm{e}_{2}$.

Figure 8 shows the total cost of the CDS comparison for all CDS construction algorithms with those of RC-CDS. From the simulation results it is observed that the total cost of the CDS of RC-CDS is 70 joules for a network size of about 400 nodes. But CCH-MCDS, E-MCDS and IPCDS algorithms have the backbone cost of the network 142J, 124J and 94J. Since in the RC-CDS, the CDS is constructed from the MIS and also the dominating nodes are selected in the succeeding rounds based on the energy level of those nodes. The CDS cost of RC-CDS is maximum up to 115 joules because the CDS cost is directly related to the energy consumption of the nodes. But in the case of other algorithms, the CDS cost of the network is between 145 to 220 joules for a network of size 600 nodes. 


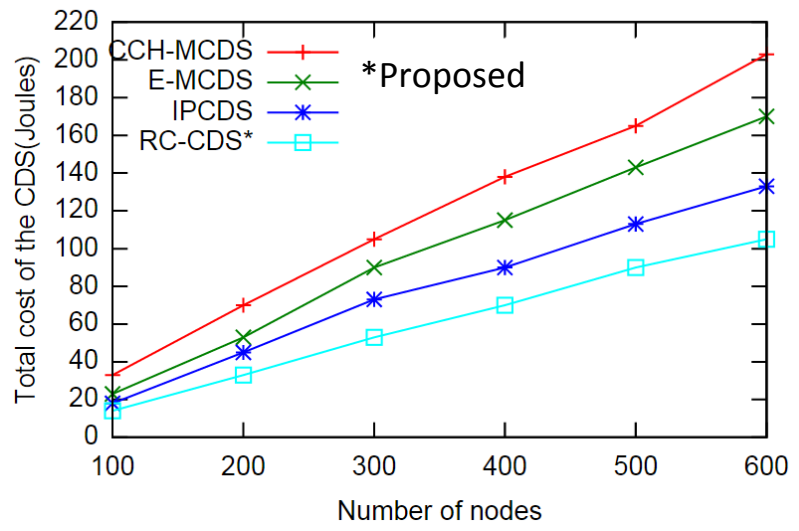

Figure 8 Total cost of the CDS

Figure 9 shows the comparison of the size of the connected dominating set produced by the proposed algorithm RC-CDS with the other existing algorithms. The number of dominating nodes is minimum compared with those of other algorithms because the RC-CDS algorithm chooses only dominant nodes with adequate energy levels. For different network sizes, $\mathrm{CCH}-\mathrm{MCDS}$ and E-MCDS relatively form an equal number of dominators. The number of dominators of the proposed RC-CDS is 28 for 300 nodes. While other protocols have the size of dominating set between 31 and 43 nodes, in $\mathrm{CCH}-$ MCDS, the number of dominators is approximately $16 \%$ of the network size. In the case of RC-CDS, the number of dominators is approximately $9 \%$ of the network size.

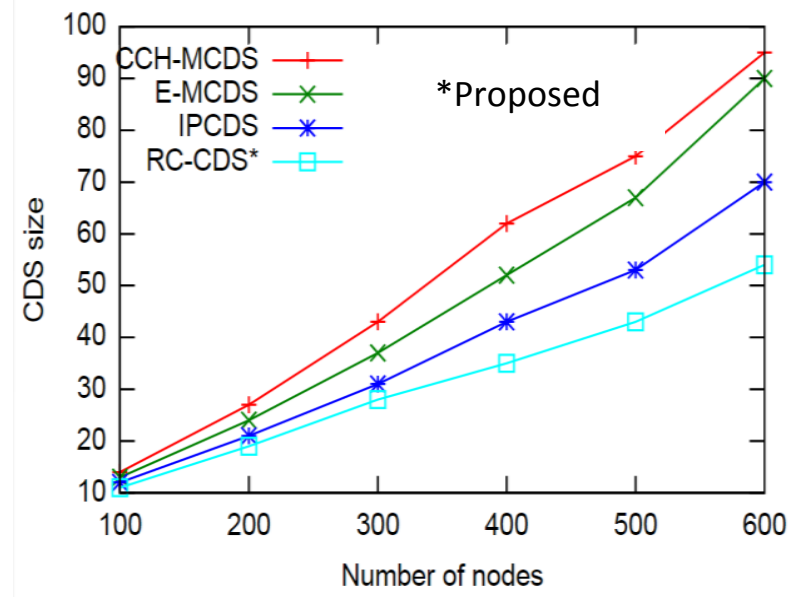

Figure 9 Comparison of CDS Size

Figure 10 shows the comparison of the average risk factor of all the CDS construction algorithms. The RC-CDS algorithm selects the dominating nodes that have the capability to send the aggregated data to the base station directly or less number of hop counts. The maximum risk of RC-CDS is $30 \%$ for a network size of 600 nodes, which is $33 \%$ lower than that of $\mathrm{CCH}-\mathrm{MCDS}$. The average risk is directly proportional to the hop count. Hence it provides optimal routing and retains the number of hops between the dominant nodes and the base station to a minimum possible level. Hence, the average risk gets decreased.

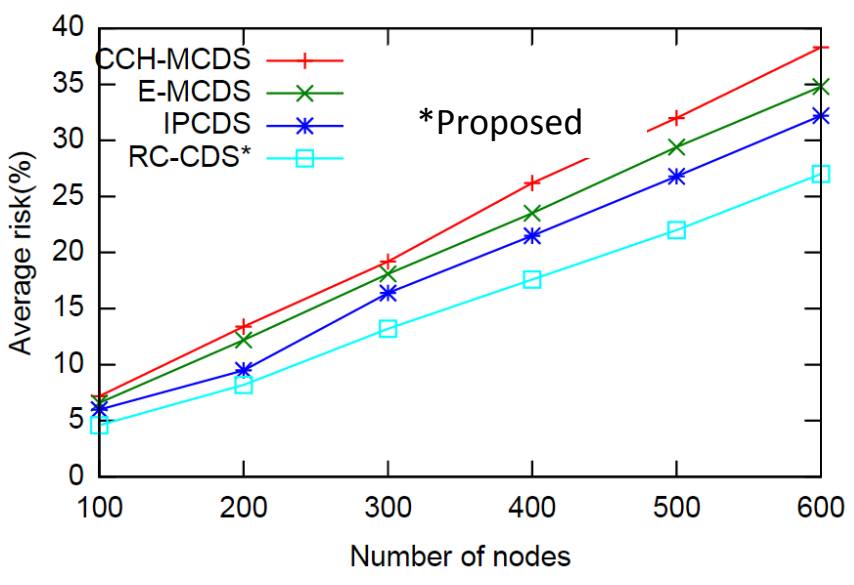

Figure 10 Comparison of Average Risk

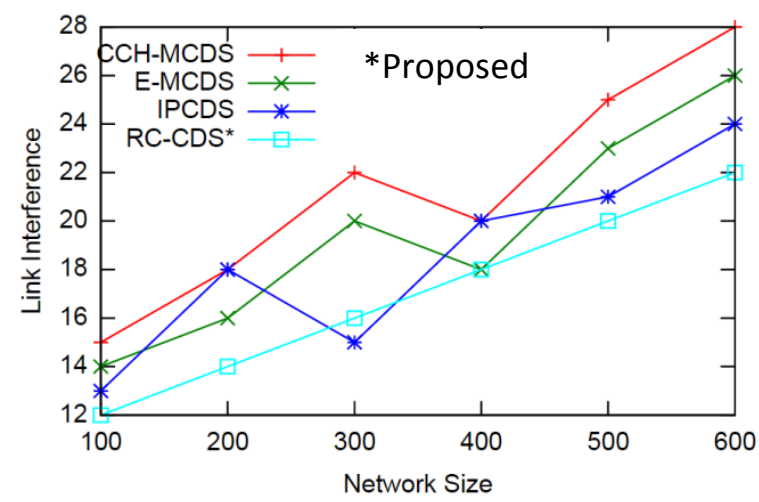

Figure 11 Comparison of Interference

Figure 11 shows the comparison of the link interference of all the CDS construction algorithms. The RC-CDS algorithm constructs a CDS with minimum size and hence the number of hops between dominant nodes and the base station is minimum. The maximum interference of RC-CDS is 22 for a network size of 600 nodes, which is $27 \%$ lower than that of CCH-MCDS. The interference is directly proportional to delivery delay. Hence it supports optimal data transmission.

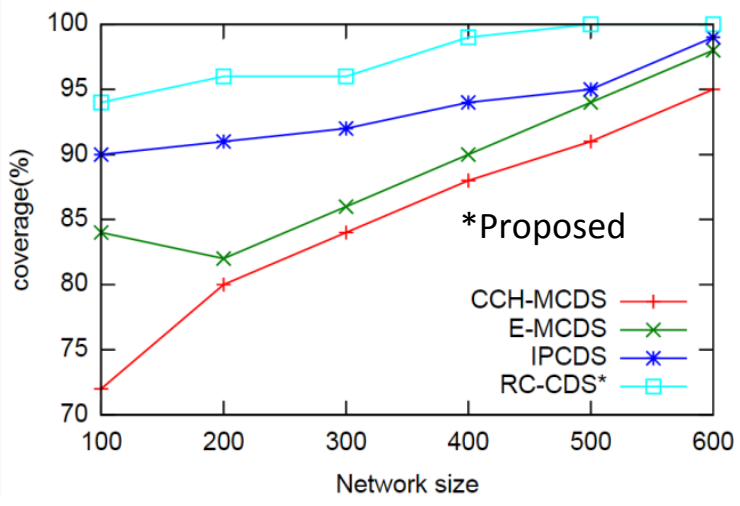

Figure 12 Coverage Vs Network Size

Figure 12 shows the results obtained regarding coverage of the network with respect to the number of nodes for different protocols. All the protocols preserve more than $70 \%$ of the coverage area by varying the network size from 100 to 600 nodes. But it is revealed that the RC-CDS preserves $100 \%$ effective coverage area when the size of the network is reached from 500 to 600 nodes. But the other protocols have only 91 to $99 \%$ of the coverage area during the network of 
size from 500 to 600 nodes. Since in the proposed RC-CDS, only dominating nodes are maintained in working mode to ensure desired sensing coverage, and other nodes are allowed to make sleep most of the time. Also a condition is imposed here that only those nodes which have a higher energy level and ensuring maximum coverage area of the network can act as dominating nodes for the succeeding rounds.

Figure 13 shows the evolution of percentage of dominant nodes round by round for all the four protocols. Some observations are in order. The number of dominant nodes varies from $45 \%$ to $75 \%$ during 100 rounds. This difference of $30 \%$ is due to the fact that the dominant nodes in the proposed scheme remain unconnected. The proposed RC-CDS has lower percentage of dominant nodes since it reduces the size of CDS during step 4 in the CDS construction phase. This step still removes some nodes in CDS. Because they act as dominant node but originally they are the connectors.

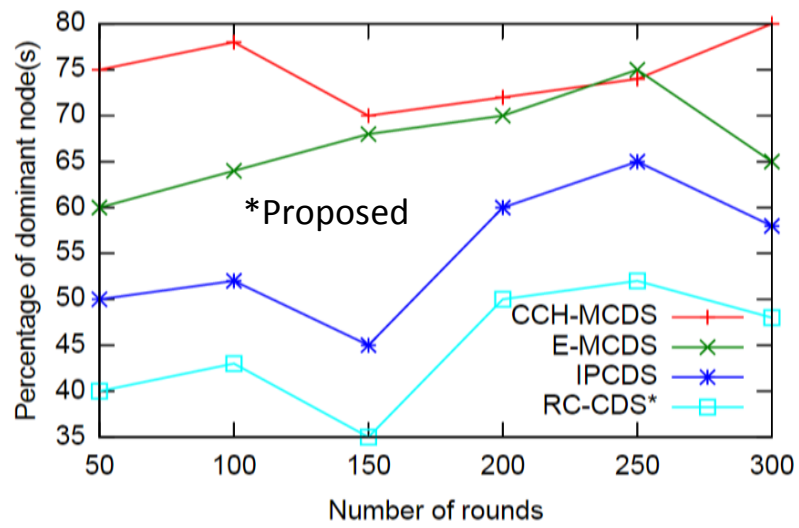

Figure 13 Dominant Nodes Vs Number of rounds

Figure 14 shows the performance of CDS size by the impact of changing the sensing range from $10 \mathrm{~m}$ to $50 \mathrm{~m}$. Each time when the sensing range increases, the network is moved from sparse network to dense network. The CDS size decreases each time sensing range increases. The sensing range is inversely proportional to the size of CDS. The increase in sensing range allows a dominator node to dominate farther nodes. It also allows a connector node to connect many dominators at the same time. Hence, the CDS size is reduced as a result of having fewer dominators and connectors. This relationship is valid for all four CDS construction algorithm. Their curves are almost identical.

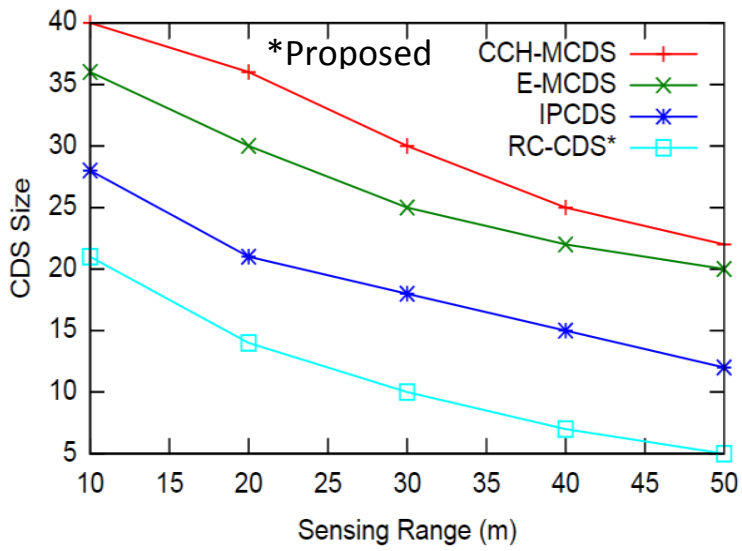

Figure 14 Sensing Range Vs CDS Size

Table 2 shows the comparison of RC-CDS with the existing CDS construction techniques for different parameters. In existing CDS construction algorithms, the total cost of the CDS is high compared to the proposed scheme. Also the size of the CDS is minimum compared to other schemes. This is due to the elimination of dominant act nodes since those nodes are connectors.

Table 2 Comparison of various coverage schemes

\begin{tabular}{|l|l|l|l|l|}
\hline Parameters & $\begin{array}{l}\text { CCH- } \\
\text { MCDS }\end{array}$ & E-MCDS & $\begin{array}{l}\text { IP- } \\
\text { CDS }\end{array}$ & RC-CDS \\
(Proposed)
\end{tabular}

\section{SUMMARY}

This chapter presents a CDS construction algorithm named Reduced Cardinality Connected Dominating Set (RC-CDS) which considers the cardinality factor as the prime criterion. It consists of four phases namely computation of maximal independent set, construction of connected dominating set, reduce the size of CDS and node self-scheduling phase. In MIS construction, a MIS is constructed from a given network graph. In CDS construction, a dominating set is created which is based on MIS. It includes the process of dominating tree and CDS queue construction and finally connectors identification for CDS. In the third phase dominant act nodes are removed which produces the minimum size CDS compared to other schemes. In the final phase of node selfscheduling, uses a dynamic reconstruction strategy of changing the dominant nodes to minimize the energy consumption of the nodes in the network.

The achievability of the proposed scheme is evaluated through performance analysis and simulation results. The results show 
that proposed scheme outperforms well compared to the existing schemes in terms of maximizes the coverage with respect to number of nodes, minimizes the average risk, reduces the size of the CDS and link interference and increases the percentage of dominant nodes with respect to number of rounds. Hence the proposed approach maintains connectivity with the help dominating set based approach in comparison to other schemes.

\section{REFERENCES}

[1] Balamurugan \& Purusothaman 2012, 'IPSD: New coverage preserving and connectivity maintenance scheme for improving lifetime of wireless sensor networks', WSEAS transactions on communications, vol.11, no.1, pp.26-36.

[2] Yiwei Wu, Feng Wang, Thai \& Yingshu Liv 2007, 'Constructing k-connected m-dominating sets in wireless sensor networks', IEEE Military Communications Conference, pp.1-7.

[3] Weili Wu, Hongwei Du, Xiaohua Jia, Yingshu Li \& Scott C-H Huang 2006, 'Minimum connected dominating sets and maximal independent sets in unit disk graphs', Journal of Theoretical Computer Science, vol.352, no.1, pp.1-7.

[4] Raihan Hazaika 2014, 'A Load Based Approach to forming a connected dominating set for an adhoc network', Thesis.

[5] Mallikarjun Avula, Seong-Moo Yoo \& Seungjin Park 2012, 'Constructing Minimum connected dominating set in mobile adhoc networks', International journal on applications of graph theory in wireless adhoc networks and sensor networks, vol.4, no.2/3, pp.15-27.

[6] Koh, Ting \& Xu 2010, 'Lower bound on the weakly connected domination number of a cycle-disjoint graph',
Australian Journal of Combinatorics, vol.46, pp.157166

[7] Rourke 1998, 'Computational Geometry in C', Cambridge university, pp.1-358.

[8] Kamrul Islam 2010, 'Energy aware techniques for certain problems in wireless sensor networks', Thesis

[9] Alim Al Islam, Chowdhury Sayeed Hyder, Humayun Kabir \& Mahmuda Naznin 2010, 'Stable Sensor Network(SSN): A dynamic clustering technique for maximizing stability in wireless sensor networks', Scientific Research in Wireless Sensor Networks, vol.2, no.7, pp.538-554.

[10] Rosana Lachowski, Marcelo Pellenz, Manoel Penna, Edgard Jamhour \& Richard Souza 2015, 'An efficient distributed algorithm for constructing spanning trees in wireless sensor networks', Journal of Sensors, vol.15, no.1, pp.1518-1536.

[11] Misra, Hong, Xue \& Tang 2010, 'Constrained relay node placement in wireless sensor networks: Formulation and approximations', IEEE/ACM Transaction Networks, vol.18, no.2, pp.434-447.

[12] Qiang Tang, Kun Yang, Ping Li, Jianming Zhang, Yuansheng Luo \& Bing Xiong 2012, 'An energy efficient MCDS construction algorithm for wireless sensor networks', EURASIP Journal on Wireless Communications and Networking, vol83, pp.1-15.

[13] Shi Ting-jun, Shi Xu \& Fang Xu-ming 2014, 'A Virtual Backbone Construction Algorithm Based on Connected Dominating Set in Wireless Sensor Networks', International Conference on Computer, Communications and Information Technology, pp.156-159. 